\title{
Publisher Correction to: 18F-florbetaben positron emission tomography detects cardiac involvement in systemic AA amyloidosis
}

\author{
Maria Papathanasiou ${ }^{1,2}$ - Alexander Carpinteiro ${ }^{2,3,4} \cdot$ Tim Hagenacker $^{2,5} \cdot$ Ken Herrmann $^{2,6} \cdot$ Tienush Rassaf $^{1,2}$. \\ Christoph Rischpler ${ }^{2,6} \cdot$ Peter Luedike $^{1,2}$
}

Published online: 15 June 2020

(C) Springer-Verlag GmbH Germany, part of Springer Nature 2020

\section{Publisher's Correction to: European Journal of Nuclear Medicine and Molecular Imaging https://doi.org/10.1007/s00259-020-04861-4}

The correct title for this paper should be: $18 \mathrm{~F}$-florbetaben positron emission tomography detects cardiac involvement in systemic AA amyloidosis

The Publisher regret's this error and the original article has been corrected.

This article is part of the Topical Collection on Erratum.

The online version of the original article can be found at https://oi.org/ $10.1007 / \mathrm{s} 00259-020-04861-4$

Maria Papathanasiou

Maria.Papathanasiou@uk-essen.de

1 Department of Cardiology and Vascular Medicine, West German, Heart and Vascular Center, University Hospital Essen,

Hufelandstrasse 55, 45147 Essen, Germany

2 West German Amyloidosis Center, University Hospital Essen, Hufelandstrasse 55, 45147 Essen, Germany

3 Department of Hematology, University Hospital Essen, Hufelandstrasse 55, 45147 Essen, Germany

4 Department of Molecular Biology, University of Duisburg-Essen, Hufelandstrasse 55, 45147 Essen, Germany

5 Department of Neurology, University Hospital Essen, Hufelandstrasse 55, 45147 Essen, Germany

6 Department of Nuclear Medicine, University Hospital Essen, Hufelandstrasse 55, 45147 Essen, Germany 